\title{
SMALL FINITE DEFORMATIONS OF ELASTIC DIELECTRICS*
}

\author{
BY \\ MANOHAR SINGH** \\ North Carolina State University at Raleigh
}

1. Introduction. As in finite elasticity theory, there are certain problems in the theory of finite deformations of elastic dielectrics which can be solved exactly by the inverse method. The deformation and the electric field are prescribed at the outset and it is then verified that the resulting state can be supported without mechanical body force or distributed charge in every homogeneous, isotropic, incompressible, elastic dielectric. Singh and Pipkin [1] have described all such possible combinations and call them controllable states of these materials. Since these states do not require any detailed knowledge of the form of the stored-energy function for the material, they could be used in an experimental determination of the stored-energy function. While there is a moderately large number of controllable states for incompressible, dielectrics [1], Singh [2] has proved that if the dielectric considered is compressible, then the only controllable states possible are the combinations of pure homogeneous deformations and the uniform electric fields. For a problem in which the displacement or the electric field, prescribed initially, are other than those in controllable states, progress towards the solution is sometimes possible if certain specific forms of the stored-energy function are made available.

In finite elasticity theory, the development of small but finite deformations was consistently developed by Murnaghan [3] and later by Rivlin [4]. In the present paper we carry out a similar investigation for elastic dielectrics. The stored-energy is assumed to be a function of the deformation gradients and the total electric field present. Constitutive relations relating stress, strain, electric field, and the dielectric displacement field are suggested. The special polynomial forms of the stored-energy function of an initially homogeneous, isotropic, elastic dielectric are developed for small finite deformations and weak electric fields. The application of the corresponding approximate constitutive equations is then illustrated by obtaining the solution of a problem which cannot be solved on the basis of a completely arbitrary stored-energy function.

Certain polynomial approximations have also been considered by Toupin [5], and Eringen [6]. No analytical solutions based on such approximations have been obtained as far as we know. The approximations arrived at in this paper are different from those in [5] and [6]. The analysis in this presentation assumes the total electric field rather than polarization as the independent variable. Also, another difference is that we do not decompose the stress, the electric field, or the dielectric displacement field into sums of various parts. When the electrical effects are not present, it is shown that the approximate forms of the stored-energy function reduce to the well-known strainenergy forms of Murnaghan [3], Mooney-Rivlin materials [7], and Neo-Hookean solids [8], in the theory of finite deformations of elastic solids.

In Secs. 2 and 3 the basic equations of the theory of elastic dielectrics are restated

*Received August 23, 1966.

**Presently at Simon Fraser University, B. C., Canada 
from [1]. This theory we believe is, in essence, equivalent to that formulated by Toupin [5], [9], where the discussion of fundamental aspects is also given.

Following the scheme suggested by Rivlin [10] in finite elasticity theory, we derive the first order and second order approximate polynomial forms of the stored-energy function in Secs. 4-6. These special forms, although valid only for small strains and weak electric fields, nevertheless admit arbitrary rotations. These forms are further specialized to cover the case of classical electrostriction when rotations are also small.

In the remainder of the paper, we examine the problem of simultaneous extensiona simple shear parallel to the axis and a simple shear about the axis-of a long circular tube of a homogeneous, isotropic, incompressible, elastic dielectric material in the presence of a radial electric field. Singh and Pipkin [1] have shown that if the functional dependence of the stored-energy function is arbitrary, the deformation and the field described above cannot together be supported without mechanical body force or charge distribution. However, it is demonstrated in this presentation that if attention is confined to small finite deformations and weak electric fields, then the problem can be solved. Closed form expressions for shear functions and the surface tractions necessary to support the prescribed combination of deformation and the electric field are determined within the first order approximation.

Finally, we may point out that with the procedure developed, because of its simplicity, solutions to many other problems can be obtained, but have not been included here since the method of treatment is now made clear.

We use the familiar suffix notation, summation convention, and Cartesian coordinate systems unless specified otherwise.

2. Continuum electrostatics. We reproduce here those basic equations of continuum electrostatics and mechanics which are independent of the composition of the material media that may be involved.

The macroscopic electric field $E_{i}$ is conservative:

$$
E_{i, j}=E_{i, i} \text {. }
$$

In the assumed absence of distributed charge, the dielectric displacement field $D_{i}$ is solenoidal:

$$
D_{i, i}=0 \text {. }
$$

At the charge-free surface of a dielectric with outward unit normal $n_{i}$, the tangential component of $E_{i}$ and the normal component of $D_{i}$ are continuous:

$$
e_{i j k}\left(E_{j}^{(0)}-E_{i}\right) n_{k}=0, \quad\left(D_{i}^{(0)}-D_{i}\right) n_{i}=0,
$$

where $E_{i}^{(0)}$ and $D_{i}^{(0)}$ are evaluated in the outside medium, and $E_{i}$ and $D_{i}$ are evaluated inside the dielectric.

We assume that the resultant force $F_{i}$ and moment $G_{i}$ exerted on the material contained in an arbitrary volume $V$, not including gravitational or inertial forces and moments, can be expressed entirely in terms of a stress vector $t_{i}$ acting over the surface $S$ of the volume $V$, in the forms:

$$
F_{i}=\oiint_{S} t_{i} d S, \quad G_{i}=\oiint_{S} e_{i i k} x_{i} t_{k} d S .
$$

Here $x_{i}$ are Cartesian coordinates, and $G_{i}$ is the moment about the origin. The stress 
vector $t_{i}$ accounts for all electro-mechanical effects except the gravitational and mechanical body forces which will be set equal to zero in the work to follow. We specifically exclude surface couples and body couples.

On the basis of the assumptions (2.4), one obtains as in continuum mechanics,

$$
t_{i}=\sigma_{i j} n_{i} \text { on the surface } S,
$$

that the stress matrix is symmetric,

$$
\sigma_{i i}=\sigma_{i i},
$$

and that the differential form of the equation of translational equilibrium, when gravitational or inertial forces are neglected, is

$$
\sigma_{i j, i}=0 .
$$

If $T_{i}$ represents the mechanical force per unit area applied on the boundary of the dielectric body, then

$$
T_{i}=\left(\sigma_{i j}-\sigma_{i j}^{(0)}\right) n_{i},
$$

where the superscript zero carries the same meaning as in (2.3). In the absence of electrical effects, at the surface of a body, one ordinarily takes the stress $\sigma_{i i}^{(0)}$ in the surrounding free space to be zero. In the present case, however, there will be a nonzero Maxwell stress everywhere.

3. Constitutive equations. In free space,

$$
D_{i}=\epsilon E_{i},
$$

$\epsilon$ being the physical constant for free space.

The stress $\sigma_{i j}^{(0)}$ in free space is the Maxwell stress defined by

$$
\sigma_{i j}=\epsilon E_{i} E_{i}-(\epsilon / 2) E_{k} E_{k} \delta_{i j} .
$$

Henceforth the dielectric bodies that we consider are surrounded by a charge free medium obeying constitutive equations of the forms (3.1) and (3.2). Apparently, the stress (3.2) satisfies (2.7) identically by virtue of (2.1), (2.2), and (3.1).

The deformation of a material dielectric body may be described by specifying coordinates $x_{i}\left(X_{1}, X_{2}, X_{3}\right)$, in the deformed state, of the generic particle which initially was located at $X_{i}$ in the undeformed state, all coordinates being measured with respect to a single fixed rectangular Cartesian system $x$. The elastic dielectric bodies to which we confine our discussion are those for which the stored-energy $W$ as a function of deformation gradients $\partial x_{i} / \partial X_{i}$ and the electric field $E_{i}$ exists, resulting in the constitutive relations:

$$
\sigma_{i i}=\rho \frac{\partial W}{\partial\left(\partial x_{i} / \partial X_{k}\right)} \frac{\partial x_{i}}{\partial X_{k}}+\rho \frac{\partial W}{\partial E_{i}} E_{i}
$$

and

$$
D_{i}=\partial W / \partial E_{i},
$$

where $\rho$ is the mass density measured in the deformed configuration of the dielectric. Constitutive relations somewhat equivalent to (3.3) and (3.4) were originally derived by Toupin [5] through a principal of virtual work by postulating the existence of a 
stored-energy function. In a latter paper [9], a different analysis was employed yielding the same constitutive equations. The theory suggested here in the form of relations (3.3) and (3.4) differs formally from Toupin's theory in that we take the electric field $E_{i}$, rather than the polarization, as the independent variable. No attempt has been made in the present paper at the formulation of an energy principle which should yield the relations (3.3) and (3.4).

Further restrictions upon the form of $W$ can be obtained from the requirement that if the dielectric body is subjected to a rigid rotation, together with the field $E_{i}$, then the force system will undergo the same rotation. Under this assumption it follows that the stored-energy function must be expressible in the form

$$
W=W\left(\left(\partial x_{k} / \partial X_{p}\right)\left(\partial x_{k} / \partial X_{\imath}\right),\left(\partial x_{p} / \partial X_{\imath}\right) E_{p}\right) .
$$

If the medium is holohedral isotropic in its undeformed field free state, then the stored-energy $W$ can be further restricted to be a function of the six scalar invariants [11]:

$$
W=W\left(I_{1}, I_{2}, I_{3}, I_{4}, I_{8}, I_{6}\right) \text {, }
$$

where

$$
\begin{aligned}
& I_{1}=g_{i i}, \quad I_{2}=\frac{1}{2}\left[g_{i i} g_{i j}-g_{i i} g_{i i}\right], \quad I_{3}=\operatorname{Det}\left|g_{i j}\right|, \\
& I_{4}=E_{i} E_{i}, \quad I_{5}=E_{i} g_{i j} E_{j}, \quad I_{6}=E_{i} g_{i k} g_{k j} E_{i} .
\end{aligned}
$$

The matrix $g_{i j}$ is the Finger strain tensor defined by

$$
g_{i i}=\left(\partial x_{i} / \partial X_{k}\right)\left(\partial x_{i} / \partial X_{k}\right) .
$$

The substitution of $W$ given by (3.6) into the constitutive equations (3.3) and (3.4) gives

$$
\begin{aligned}
\sigma_{i i}=\frac{2}{\left(I_{3}\right)^{1 / 2}}\left[\left(\frac{\partial W}{\partial I_{1}}\right.\right. & \left.+I_{1} \frac{\partial W}{\partial I_{2}}\right) g_{i i}-\frac{\partial W}{\partial I_{2}} g_{i i}^{2}+I_{3} \frac{\partial W}{\partial I_{3}} \delta_{i i} \\
& +\frac{\partial W}{\partial I_{4}} E_{i} E_{i}+\frac{\partial W}{\partial I_{5}}\left(g_{i k} E_{i} E_{k}+g_{i k} E_{k} E_{i}\right) \\
& \left.+\frac{\partial W}{\partial I_{6}}\left(g_{i k}^{2} E_{i} E_{k}+g_{i k}^{2} E_{i} E_{k}\right)+\frac{\partial W}{\partial I_{6}} g_{i k} g_{i l} E_{b} E_{l}\right],
\end{aligned}
$$

and

$$
D_{i}=2\left(\frac{\partial W}{\partial I_{4}} \delta_{i i}+\frac{\partial W}{\partial I_{5}} g_{i j}+\frac{\partial W}{\partial I_{6}} g_{i i}^{2}\right) E_{i} .
$$

We use $g_{i j}^{2}$ to denote the $i j$-component of the square of the matrix $\mathbf{g}$.

Considering the stress and the dielectric displacement field as functions of the deformation gradients and the electric field, Singh and Pipkin [1] formulated relations equivalent to (3.9) and (3.10) with the difference that they did not use the expressions for response coefficients in terms of derivatives of the stored-energy.

4. Approximate theories. Assuming the stored-energy function $W$ in (3.6) to be a polynomial function of its arguments, we may without loss of generality write

$$
W=\sum_{\alpha, \beta, \gamma, \delta, \lambda, \mu} A_{\alpha \beta \gamma \delta \lambda_{\mu}}\left(I_{1}-3\right)^{\alpha}\left(I_{2}-3\right)^{\beta}\left(I_{3}-1\right)^{\gamma} I_{4}^{\delta} I_{5}^{\lambda} I_{6}^{\mu},
$$

where $A_{\alpha \beta \gamma \delta \lambda_{\mu}}$ are constants of the material. It is readily noted from (4.1) that if the 
medium is undeformed and field free,

$$
I_{1}-3=I_{2}-3=I_{3}-1=I_{4}=I_{3}=I_{6}=0 \text {. }
$$

Let $e_{1}, e_{2}, e_{3}$ denote the principal extensions at a point $P$ of the dielectric body and $E_{1}, E_{2}, E_{3}$ the components of the electric field referred to the principal directions of strain at $P$. The invariants (3.7) then become

$$
\begin{aligned}
& I_{1}=\left(1+e_{1}\right)^{2}+\left(1+e_{2}\right)^{2}+\left(1+e_{3}\right)^{2}, \quad I_{3}=\left(1+e_{1}\right)^{2}\left(1+e_{2}\right)^{2}\left(1+e_{3}\right)^{2}, \\
& I_{2}=\left(1+e_{1}\right)^{2}\left(1+e_{2}\right)^{2}+\left(1+e_{2}\right)^{2}\left(1+e_{3}\right)^{2}+\left(1+e_{3}\right)^{2}\left(1+e_{1}\right)^{2}, \quad I_{4}=E_{i} E_{i}, \\
& I_{5}=\left(1+e_{1}\right)^{2} E_{1}^{2}+\left(1+e_{2}\right)^{2} E_{2}^{2}+\left(1+e_{3}\right)^{2} E_{3}^{2}, \\
& I_{6}=\left(1+e_{1}\right)^{4} E_{1}^{2}+\left(1+e_{2}\right)^{4} E_{2}^{2}+\left(1+e_{3}\right)^{4} E_{3}^{2} .
\end{aligned}
$$

If $I_{1}-3, I_{2}-3, I_{3}-1, I_{4}, I_{5}$, and $I_{6}$, are sufficiently small, we may approximate $W$, to any desired order in the principal extensions and powers of the electric field components, by neglecting terms above an appropriate degree in the series expansion (4.1). Any such approximate form of $W$ will be invariant to all rigid rotations simultaneously of the dielectric body and the electric field. It is evident, however, that the quality of the approximation sought will depend upon the magnitude of the physical constants $A_{\alpha \beta \gamma \delta \lambda_{\mu}}$.

5. First approximation. Suppose the principal extensions $e_{i}$ are small and the electric field $E_{i}$ sufficiently weak. We may define the first approximation as one in which we retain in $W$ all terms involving principal extensions $e_{i}$ to a lower degree than the third, the terms containing the field components $E_{i}$ to a degree lower than the fourth, and the product terms only of the type $e_{i} E_{i}^{2}$. In order to obtain the form of $W$ for such an approximation, we will have to retain twenty-two terms in the series expansion (4.1). However, we can arrive at the same approximation involving fewer terms by introducing a new set of six mutually independent scalar invariants:

$$
\begin{aligned}
& J_{1}=I_{1}-3, \quad J_{2}=\left(I_{2}-3\right)-2\left(I_{1}-3\right), \\
& J_{3}=\left(I_{3}-1\right)-\left(I_{2}-3\right)+\left(I_{1}-3\right), \\
& J_{4}=I_{4}, \quad J_{5}=I_{5}-I_{4}, \quad J_{6}=I_{6}-2\left(I_{5}-I_{4}\right)-I_{4} .
\end{aligned}
$$

In place of (4.1) we now have

$$
W=\sum_{\alpha, \beta, \gamma, \delta, \lambda, \mu} B_{\alpha \beta \gamma \delta \lambda \mu} J_{1}^{\alpha} J_{2}^{\beta} J_{3}^{\gamma} J_{4}^{\delta} J_{5}^{\lambda} J_{6}^{\mu},
$$

where $B$ 's are physical constants of the material.

In view of (4.2), (5.1), and the statement of the first approximation, the following form for the stored energy function is obtained from (5.2):

$$
W=a_{0}+a_{1} J_{1}+a_{2} J_{2}+a_{3} J_{1}^{2}+a_{4} J_{4}+a_{5} J_{5}+a_{6} J_{1} J_{4} \text {. }
$$

To facilitate the writing, $a$ 's are being used here to denote the $B$ 's in (5.2). Since we may, without loss of generality, assume vanishing stored-energy and stress in the initial undeformed field free state, Eqs. (5.3) and (3.9) yield $a_{0}=a_{1}=0$, so that (5.3) reduces to

$$
W=a_{2} J_{2}+a_{3} J_{1}^{2}+a_{4} J_{4}+a_{5} J_{5}+a_{6} J_{1} J_{4} \text {. }
$$

We may remark here that when electrical effects are absent, the last three terms 
in (5.4) drop out leaving behind the first approximate form of the strain-energy function in the theory of finite elasticity [10].

Introduction of (5.4) into (3.9) and (3.10) results in the following constitutive relations:

$$
\begin{aligned}
\sigma_{i j}=2 \rho\left[\left[a_{2}+\left(a_{2}+2 a_{3}\right)\right.\right. & \left.J_{1}+a_{6} J_{4}\right] g_{i j} \\
& \left.-a_{2} g_{i j}^{2}+\left(a_{4}-a_{5}\right) E_{i} E_{i}+a_{5}\left(g_{i k} E_{k} E_{i}+g_{i k} E_{k} E_{i}\right)\right],
\end{aligned}
$$

and

$$
D_{i}=2\left[\left(a_{4}-a_{5}\right) \delta_{i i}+a_{5} g_{i j}\right] E_{i} .
$$

In the expression (5.4) for $W$ if we further neglect terms of degree higher than the second in displacement gradients $\partial u_{i} / \partial X_{i}$ and the field components $E_{i}$, and product terms of order higher than $\left(\partial u_{i} / \partial X_{i}\right) E_{k}^{2}$, then we obtain

$$
W=2 a_{2}\left(e_{i i} e_{i j}-e_{i j} e_{i j}\right)+4 a_{3} e_{i i} e_{i j}+a_{4} E_{i} E_{i}+2 a_{5} e_{i j} E_{i} E_{i}+2 a_{6} e_{i i} E_{k} E_{k},
$$

where $e_{i j}$ is the classical linear strain tensor defined by

$$
e_{i i}=\frac{1}{2}\left(\partial u_{i} / \partial X_{i}+\partial u_{i} / \partial X_{i}\right),
$$

with

$$
u_{i}=x_{i}-X_{i} .
$$

One may then designate $W$ given by (5.7) as the stored-energy function for the classical coupled theory of electrostriction. Both the approximate forms (5.4) and (5.7) are valid only for small strains and weak electric fields. However, it is essential to note the distinction between these two types of approximations. Whereas the form (5.4) admits arbitrary rotations, only infinitesimal rotations, even rigid, are permissible in the application of (5.7).

6. Second approximation. A second approximation can be arrived at by retaining in the expansion (5.2) for $W$ terms up to and including third powers in principal extensions $e_{i}$ and field components $E_{i}$, and product terms up to and including $e_{i}^{2} E_{i}^{2}$. To this end the corresponding expression for $W$ is

$$
\begin{aligned}
W=a_{2} J_{2} & +a_{3} J_{1}^{2}+a_{4} J_{4}+a_{5} J_{5}+a_{6} J_{1} J_{4}+a_{7} J_{1}^{3} \\
& +a_{8} J_{1} J_{2}+a_{8} J_{3}+a_{10} J_{2} J_{4}+a_{11} J_{1}^{2} J_{4}+a_{12} J_{6}+a_{13} J_{1} J_{5},
\end{aligned}
$$

where $a$ 's, as before, are material constants. In writing (6.1), it is assumed that $W$ and stresses vanish in the undeformed field free state.

We note that when the electric field is not present, then (6.1) reduces to the wellknown Murnaghan's form [3] in the theory of finite deformations of elastic solids.

The corresponding approximate form for $W$ in the classical sense when rotations are also small can now be obtained by further neglecting in (6.1) those terms which are of a degree higher than the third in the quantities $\partial u_{i} / \partial X_{i}$ or $E_{i}$ as well as product terms of order higher than $\left(\partial u_{i} / \partial X_{i}\right)^{2} E_{k}^{2}$. This approximation yields

$$
\begin{gathered}
W=2\left(a_{2}+2 a_{3}\right)\left(\frac{\partial u_{i}}{\partial X_{i}}\right)^{2}-a_{2}\left(\frac{\partial u_{i}}{\partial X_{k}} \frac{\partial u_{i}}{\partial X_{k}}+\frac{\partial u_{i}}{\partial X_{k}} \frac{\partial u_{k}}{\partial X_{i}}\right)+2\left(a_{9}-a_{2}\right) \frac{\partial u_{i}}{\partial X_{i}} \frac{\partial u_{i}}{\partial X_{k}} \frac{\partial u_{i}}{\partial X_{k}} \\
+2\left(a_{2}+2 a_{3}-a_{8}-a_{9}\right) \frac{\partial u_{i}}{\partial X_{i}} \frac{\partial u_{i}}{\partial X_{k}} \frac{\partial u_{i}}{\partial X_{k}}+4\left(a_{8}+2 a_{7}+\frac{1}{3} a_{9}\right)\left(\frac{\partial u_{k}}{\partial X_{k}}\right)^{3}
\end{gathered}
$$




$$
\begin{aligned}
& -2\left(a_{8}+a_{9}\right) \frac{\partial u_{i}}{\partial X_{i}} \frac{\partial u_{i}}{\partial X_{k}} \frac{\partial u_{k}}{\partial X_{i}}+\frac{2}{3} a_{9} \frac{\partial u_{i}}{\partial X_{i}} \frac{\partial u_{i}}{\partial X_{k}} \frac{\partial u_{k}}{\partial X_{i}}+a_{4} E_{i} E_{i}+2 a_{5} \frac{\partial u_{i}}{\partial X_{i}} E_{i} E_{i} \\
& +2 a_{6} \frac{\partial u_{i}}{\partial X_{i}} E_{k} E_{k}+\left(2 a_{10}+4 a_{11}\right)\left(\frac{\partial u_{i}}{\partial X_{i}}\right)^{2} E_{k} E_{k}-a_{10} \frac{\partial u_{i}}{\partial X_{i}} \frac{\partial u_{i}}{\partial X_{i}} E_{k} E_{k} \\
& +\left(2 a_{5}+a_{12}\right) \frac{\partial u_{i}}{\partial X_{k}} \frac{\partial u_{i}}{\partial X_{k}} E_{i} E_{i}+\left(2 a_{6}-a_{10}\right) \frac{\partial u_{i}}{\partial X_{i}} \frac{\partial u_{i}}{\partial X_{i}} E_{k} E_{k}+2 a_{12} \frac{\partial u_{i}}{\partial X_{k}} \frac{\partial u_{k}}{\partial X_{i}} E_{i} E_{i} \\
& +a_{12} \frac{\partial u_{k}}{\partial X_{i}} \frac{\partial u_{k}}{\partial X_{i}} E_{i} E_{i}+2 a_{13} \frac{\partial u_{k}}{\partial X_{k}}\left(\frac{\partial u_{i}}{\partial X_{i}}+\frac{\partial u_{i}}{\partial X_{i}}\right) E_{i} E_{i}
\end{aligned}
$$

7. Incompressible dielectric. If, in addition to being homogeneous and isotropic, the elastic dielectric considered is incompressible, the third invariant of strain $I_{3}$ is unity in all deformations, and is accordingly not a variable. The stored-energy $W$ is then a function only of five invariants $I_{1}, I_{2}, I_{4}, I_{3}$, and $I_{6}$, defined in (3.7).

In terms of principal extensions $e_{i}$, the constraint of no volume change according to (4.2) demands

$$
\left(1+e_{1}\right)\left(1+e_{2}\right)\left(1+e_{3}\right)=1 \text {. }
$$

Here it is convenient to introduce a new scalar invariant $J_{2}^{\prime}$ :

$$
J_{2}^{\prime}=J_{2}-J_{1} \text {. }
$$

For the incompressible case therefore, we have instead of (5.2) the following polynomial expansion for $W$ :

$$
W=\sum_{\alpha, \beta, \delta, \lambda, \mu} B_{\alpha \beta \delta \lambda \mu} J_{1}^{\alpha} J_{2}^{\prime \beta} J_{4}^{\delta} J_{5}^{\lambda} J_{6}^{\mu} \text {. }
$$

With (7.1), it follows from (3.7), (4.2), (5.1), and (7.2) that the invariants $J_{1}$ and $J_{2}^{\prime}$ are of second and third orders, respectively, in the principal extensions. Hence, within the formulation of the first approximation for small finite deformations, defined in Sec. 5, the appropriate stored-energy-function corresponding to (5.4) is given by

$$
W=b_{1} J_{1}+b_{2} J_{4}+b_{3} J_{5}
$$

where $b$ 's are physical constants of the material.

For the second approximation described in Sec. 6, the form of $W$ that corresponds to $(6.1)$ is

$$
W=b_{1} J_{1}+b_{2} J_{4}+b_{3} J_{5}+b_{4} J_{2}^{\prime}+b_{5} J_{1} J_{4}+b_{6} J_{6} \text {. }
$$

It may be observed that in the absence of electrostatic effects, (7.4) and (7.5) reduce to the forms which in finite elasticity theory describe the well-known Neo-Hookean [8] and Mooney-Rivlin [7] materials, respectively.

In conservative systems, a pressure $p$ arises as a reaction to the constraint of no volume change. Bearing this in mind, substitution of (7.4) in (3.9) and (3.10) leads to the following constitutive relations of the first approximation:

$$
\begin{gathered}
\sigma_{i j}=-p \delta_{i j}+C_{1} g_{i i}+C_{2} E_{i} E_{i}+C_{3}\left(g_{i k} E_{k} E_{i}+g_{i k} E_{k} E_{i}\right), \\
D_{i}=C_{2} E_{i}+C_{3} g_{i j} E_{j},
\end{gathered}
$$

where $p$ is an arbitrary pressure and $C$ 's are the physical constants of the dielectric medium. 
8. Simultaneous extension and shear of a cylindrical annulus. We consider a long tube of homogeneous, isotropic, incompressible, elastic dielectric with initial internal radius $a$ and external radius $b$. The tube is subjected to a small finite deformation in the presence of a weak electric field. The particle originally located at the point $(R, \theta, Z)$ in cylindrical polar coordinates moves to the position $(r, \theta, z)$ given by

$$
r=\lambda R, \quad \theta=\theta+\phi(R), \quad z=Z / \lambda^{2}+w(R) .
$$

The above deformation is a simple extension of extension ratio $1 / \lambda^{2}$, a simple shear of the tube in which each point moves through a distance $w$ parallel to the axis and through an angle $\phi$ about the axis, both $\phi$ and $w$ dependent only on the radial position of the point. It is verified readily that the mapping (8.1) is volume preserving.

We suppose that a radial electric field is imposed by placing the tube between the plates of a coaxial cylindrical condenser. In view of (2.1) the radial field has to be of the type

$$
E_{r}=E_{r}(r), \quad E_{\theta}=E_{\imath}=0 \text { inside the dielectric, }
$$

and

$$
E_{r}^{(0)}=E_{r}^{(0)}(r), \quad E_{\theta}^{(0)}=E_{s}^{(0)}=0 \text { in the surrounding medium. }
$$

Here, as in all subsequent analysis, we shall refer to physical components in cylindrical coordinate system.

The problem proposed here is to determine $E_{r}(r), E_{r}^{(0)}(r), \phi(R), w(R)$, and the necessary surface tractions such that the deformation (8.1) can be supported without mechanical body force or distributed charge in the presence of the electric field given by (8.2) and (8.3).

In the absence of electrostatic effects, the deformation (8.1) has been studied by Rivlin [12], and Singh and Pipkin [13]. More recently, in the theory of elastic dielectrics, Singh and Pipkin [1] have shown that if the functional form of the stored-energy is regarded as arbitrary, then the deformation (8.1) can be supported without mechanical body force or distributed charge if and only if

$$
\phi(R)=B \log R, \quad w(R)=0,
$$

and the electric field is axial and uniform. However, we intend to show that if attention is confined to small finite deformations permitting the application of constitutive relations (7.6) and (7.7), a closed form solution can be obtained for $\phi(R), w(R), E_{r}(r)$, and $E_{r}^{(0)}(r)$. The surface tractions that can support (8.1) in the presence of field (8.2) and (8.3) can then be calculated.

With (8.1), the strain from (3.8) is

$$
\begin{array}{lll}
g_{r r}=\lambda^{2}, & g_{\theta \theta}=\lambda^{2}+r^{2} \phi^{\prime 2}, & g_{z z}=\frac{1}{\lambda^{4}}+w^{\prime^{2},}, \\
g_{r \theta}=\lambda r \phi^{\prime}, & g_{\theta z}=r \phi^{\prime} w^{\prime}, & g_{r z}=\lambda w^{\prime},
\end{array}
$$

where primes indicate differentiation with respect to $R$. To obtain the components of flux inside the dielectric, we substitute from (8.2) and (8.5) into (7.7):

$$
D_{r}=\left(C_{2}+C_{3} \lambda^{2}\right) E_{r}, \quad D_{\theta}=C_{3} \lambda r \phi^{\prime} E_{r}, \quad D_{z}=C_{3} \lambda w^{\prime} E_{r} .
$$

Outside the dielectric, the flux components from (3.1) are simply $1 / \epsilon$ times those of 
the electric field (8.3). The Maxwell condition (2.2) that the flux be solenoidal both inside and outside the dielectric now provides

$$
E_{r}=K / r, \quad E_{r}^{(0)}=L / r,
$$

where $K$ and $L$ are any constants. By virtue of the continuity condition (2.3a), $L=\left(C_{2}+C_{3} \lambda^{2}\right)(K / \epsilon)$. In place of (8.2) and (8.3) therefore, we now have

$E_{r}=K / r, \quad E_{0}=E_{s}=0 ; \quad E_{r}^{(0)}=\left(C_{2}+C_{3} \lambda^{2}\right) K / \epsilon r, \quad E_{\theta}^{(0)}=E_{*}^{(0)}=0$.

It is immediately clear that the field (8.8) meets the continuity condition (2.3b).

The corresponding stresses follow from using (8.5) and (8.8a) into (7.6), and appear as

$$
\begin{array}{ll}
\sigma_{r r}=-p+C_{1} \lambda^{2}+\left(C_{2}+2 C_{3} \lambda^{2}\right) \frac{K^{2}}{r^{2}}, & \sigma_{\theta \theta}=-p+C_{1}\left(\lambda^{2}+r^{2} \phi^{\prime 2}\right), \\
\sigma_{z s}=-p+C_{1}\left(\frac{1}{\lambda^{4}}+w^{\prime 2}\right), & \sigma_{r \theta}=\left(C_{1}+C_{3} \frac{K^{2}}{r^{2}}\right) \lambda r \phi^{\prime}, \\
\sigma_{r z}=\left(C_{1}+C_{3} \frac{K^{2}}{r^{2}}\right) \lambda w^{\prime}, & \sigma_{\theta s}=C_{1} r \phi^{\prime} w^{\prime} .
\end{array}
$$

With the stresses (8.9) depending only upon $r$, the equations of equilibrium (2.7) become

$$
\frac{\partial \sigma_{r r}}{\partial r}+\frac{1}{r}\left(\sigma_{r r}-\sigma_{\theta \theta}\right)=0, \quad \frac{\partial \sigma_{r \theta}}{\partial r}+\frac{2}{r} \sigma_{r \theta}=0, \quad \frac{\partial \sigma_{r z}}{\partial r}+\frac{1}{r} \sigma_{r s}=0 .
$$

The last two of (8.10), in conjunction with (8.9), can be integrated to yield

$$
\phi(R)=A \log \frac{R^{2}}{F(R)}+A_{1}, \quad w(R)=B \log F(R)+B_{1},
$$

where $A, B, A_{1}$, and $B_{1}$, are constants of integration, and to facilitate writing we have set

$$
F(R)=C_{1} \lambda^{2} R^{2}+C_{3} K^{2} .
$$

Suppose we have $w=\phi=0$ at the curved surface $r=\lambda b$, and $w=w_{0}, \phi=\phi_{0}$ at the curved surface $r=\lambda a$. These conditions allow us to determine the constants $A, B, A_{1}, B_{1}$, thus leading to

$$
\phi(R)=\Psi_{0} \log \frac{R^{2} F(b)}{b^{2} F(R)}, \quad w(R)=\Omega_{0} \log \frac{F(R)}{F(b)} .
$$

The constants $\Psi_{0}$ and $\Omega_{0}$ stand for

$$
\Psi_{0}=\frac{\phi_{0}}{\log \left(a^{2} F(b) / b^{2} F(a)\right)}, \quad \Omega_{0}=\frac{w_{0}}{\log (F(a) / F(b))} .
$$

The functions $\phi(R)$ and $w(R)$ having been so determined, we can now introduce (8.13) and (8.9) into the first of the equilibrium equations (8.10) to find the pressure $p$ :

$p(r)=\left(C_{2}+2 C_{3} \lambda^{2}\right) \frac{K^{2}}{2 r^{2}}-2 C_{1} \lambda^{2} \Psi_{0}^{2}\left[\log \frac{r^{2}}{C_{1} r^{2}+C_{3} K^{2}}+\frac{C_{3} K^{2}}{C_{1} r^{2}+C_{3} K^{2}}\right]+a$,

where $Q$ is a constant of integration. With pressure $p$ given by (8.15), and $\phi(R)$ and $w(R)$ given by (8.13), the stresses inside the dielectric medium are furnished by (8.9).

In the medium surrounding the dielectric, the Maxwell stresses follow by substituting the field (8.8b) into (3.2): 


$$
\begin{aligned}
& \sigma_{r r}^{(0)}=-\sigma_{\theta \theta}^{(0)}=-\sigma_{z z}^{(0)}=\left(C_{2}+2 C_{3} \lambda^{2}\right)^{2} K^{2} / 2 \epsilon r^{2}, \\
& \sigma_{r \theta}^{(0)}=\sigma_{\theta z}^{(0)}=\sigma_{r z}^{(0)}=0 .
\end{aligned}
$$

In order to support the deformation (8.1) in the field prescribed as in (8.2) and (8.3), the tractions which must be applied to the curved surfaces $r=\lambda a$ and $r=\lambda b$ are obtained from (2.8) when use is made of (8.9), (8.13), (8.15), and (8.16):

$$
\begin{array}{r}
T_{r}(r=\lambda a)=\left(C_{2}+2 C_{3} \lambda^{2}\right) \frac{K^{2}}{2 \lambda^{2} a^{2}}+2 C_{1} \lambda^{2} \Psi_{0}^{2}\left[\log \frac{\lambda^{2} a^{2}}{C_{1} \lambda^{2} a^{2}+C_{3} K^{2}}+\frac{C_{3} K^{2}}{C_{1} \lambda^{2} a^{2}+C_{3} K^{2}}\right] \\
-\left(C_{2}+2 C_{3} \lambda^{2}\right)^{2} \frac{K^{2}}{2 \epsilon \lambda^{2} a^{2}}+B,
\end{array}
$$

$T_{\theta}(r=\lambda a)=\frac{2 C_{3} K^{2} \Psi_{0}}{a^{2}}, \quad T_{2}(r=\lambda a)=\frac{2 C_{1} \lambda^{2} \Omega_{0}}{a}$,

The constants $\Psi_{0}$ and $\Omega_{0}$ above are as in (8.14). By setting the radial component $T_{\text {. }}$ in (8.17) of the applied traction to zero, the arbitrary constant $B$ can be easily evaluated. Expressions for surface tractions at the curved surface $r=\lambda b$ may be derived in a similar manner replacing $a$ by $b$ in (8.17) and (8.18). Since we do not have accurate exyressions for the fields near the edges bounded by the plane ends of the tube, we will not calculate the end tractions. However, it is readily noted from (8.9) and (8.16) that in case the tube is of finite length, normal and shearing tractions must be applied to its ends to maintain the given state.

We observe here that if either of the curved surfaces, say $r=\lambda a$, is free from applied tangential tractions, then since the Maxwell stresses $\sigma_{r \theta}^{(0)}$ and $\sigma_{r z}^{(0)}$ happen to be zero in the field considered, the stresses $\sigma_{r \theta}$ and $\sigma_{r z}$ inside the dielectric medium will also have to vanish by virtue of continuity conditions (2.8) and the last two of equilibrium equations (8.10). This, in conjunction with (8.9), would then demand $\phi^{\prime}(R)=w^{\prime}(R)=0$, as to be expected.

The case of no extension is the special case of the problem considered above when $\lambda$ is equal to unity. Physically, this special case may be demonstrated by holding the tube between two rigid coaxial cylinders with the outer cylinder kept fixed while moving the inner one along the axis of the tube as well as rotating it about the axis.

\section{REFERENCES}

[1] M. Singh, and A. C. Pipkin, Arch. Rational Mech. Anal. 21, 169 (1966)

[2] M. Singh, ZAMP 17, 449 (1966)

[3] F. D. Murnaghan, Amer. J. Math. 59, 235 (1937)

[4] R. S. Rivlin, J. Rational Mech. Anal. 2, 53 (1953)

[5] R. A. Toupin, J. Rational Mech. Anal. 5, 849 (1956)

[6] A. C. Eringen, Int. J. Engr. Sci. 1, 127 (1963)

[7] M. Mooney, J. Appl. Phys. 11, 582 (1940)

[8] R. S. Rivlin, Phil. Trans. Roy. Soc. London A 240, 459 (1948)

[9] R. A. Toupin, J. Engr. Sci. 1, 101 (1963)

[10] R. S. Rivlin, Some topics in finite elasticity, Proceedings of the First Symposium on Naval Structural Mechanics, Pergamon Press, New York, 1960

[11] A. C. Pipkin, and R. S. Rivlin, Arch. Rational Mech. Anal. 4, 129 (1959)

[12] R. S. Rivlin, Phil. Trans. Roy. Soc. London A 242, 173 (1949)

[13] M. Singh, and A. C. Pipkin, ZAMP 16, 706 (1965) 